Revista Brasileira de Agricultura Irrigada v.13, nº.4, p. 3512 - 3523, 2019

ISSN 1982-7679 (On-line)

Fortaleza, CE, INOVAGRI - http://www.inovagri.org.br

DOI: $10.7127 /$ rbai.v13n4001031

Protocolo 1031.18 - 19/10/2018 Aprovado em 26/07/2019

\title{
PRECIPITAÇÃO E EVAPOTRANSPIRAÇÃO DE REFERÊNCIA PROVÁVEIS PARA O MUNICÍPIO DE PINHAIS-PR
}

Jorge Luiz Moretti de Souza ${ }^{1}$, Daniela Jerszurki ${ }^{2}$, Mariana Vasconcelos Barroca ${ }^{3}$, Clodoveu Valdeni Trentin ${ }^{4}$, Bruno César Gurski ${ }^{4}$

\section{RESUMO}

Teve-se por objetivo no presente trabalho determinar os parâmetros estatísticos da função densidade de probabilidade (FDP) com melhor ajuste aos valores decendiais de precipitação pluvial (P) e evapotranspiração de referência (ETo) para o município de Pinhais-PR, e calcular os valores decendiais prováveis de P (75\%) e ETo (25\%). Foram consideradas as funções densidade Normal, Triangular, Gama, Exponencial e Uniforme e, para a verificação do ajustamento das funções às séries de dados, foram aplicados testes de aderência de Kolmogorov-Smirnov a 5\% de significância. Apesar dos valores decendiais da ETo terem se ajustado melhor à função densidade Normal $(51,4 \%)$ e a precipitação à função Gama $(54,1 \%)$, aproximadamente metade dos decêndios ao longo do ano ajustaram-se à outras quatro funções densidade de probabilidade, evidenciando a necessidade de se considerar outras funções em estudos climáticos visando a determinação de valores prováveis na região. Os valores de $\mathrm{ETo}_{25 \%}$ e $\mathrm{P}_{75 \%}$ indicaram entre março e agosto maior possibilidade de redução no armazenamento de água no solo e ocorrência de deficiência hídrica às plantas. Associações lineares simples possibilitam a obtenção dos valores decendiais de $\mathrm{ETo}_{25 \%}$ e $\mathrm{P}_{75 \%}$, respectivamente, com valores de $\mathrm{ETo}_{\text {médio }}$ e $\mathrm{P}_{\text {médio }}$ decendial da região de Pinhais.

Palavras-chave: função densidade de probabilidade, componentes hídricas, ajustamento.

\section{PROBABLE PRECIPITATION AND REFERENCE EVAPOTRANSPIRATION IN PINHAIS-PR}

\footnotetext{
ABSTRACT

${ }^{1}$ Professor do Programa de Pós Graduação em Ciência do solo, Universidade Federal do Paraná, Curitiba, PR, Brasil. Email: jmoretti@ufpr.br

${ }^{2}$ Pesquisadora do Wyler Department of Dryland Agriculture, French Associates Institute for Agriculture and Biotechnology of Drylands, Jacob Blaustein Institutes for Desert Research, Ben-Gurion University of the Negev, Sde Boker, Israel. E-mail: danijerszurki@gmail.com

${ }^{3}$ Doutoranda em Ciência do solo no departamento de Dryland Agriculture, Jacob Blaustein Institutes for Desert Research, Ben-Gurion University of the Negev, Sde Boker, Israel. E-mail: marivbarroca@gmail.com

${ }^{4}$ Universidade Federal do Paraná, Rua dos Funcionários, n. 1.540, CEP 80035-050 Curitiba, PR, Brazil. E-mail: brunogurski@ufpr.bre clodoveutrentin@yahoo.com.br
} 
The aim of this study was to determine the statistical parameters of the probability density function (PDF) with the best adjustment to the values of decennial rainfall precipitation (P) and reference evapotranspiration (ETo) for the city of Pinhais-PR, Brazil and to calculate the decennial values of probable P (75\%) and ETo (25\%). The density function Normal, Triangular, Gamma, Exponential and Uniform were considered. For checking the adjustment of probability density function to the data series, the Kolmogorov-Smirnov tests at 5\% probability was applied. Despite decennial ETo values have adjusted better to the Normal density function $(51,4 \%)$ and the precipitation to the Gamma function $(54,1 \%)$, approximately half of the periods of ten days throughout the year, also fit on other four density functions. Thus, showing the need to consider other functions for climate studies aiming to determine probable values in the region. The $\mathrm{ETo}_{25 \%}$ and $\mathrm{P}_{75 \%}$ values, suggest high possibility of lower water storage on soil and occurrence of water deficiency to plants on the period between march and august. Simple linear association enables to obtain the decennial values of $\mathrm{ETo}_{25 \%}$ and $\mathrm{P}_{75 \%}$ with values of $\mathrm{ETo}_{\text {mean }}$ e $\mathrm{P}_{\text {mean }}$, respectively, for the region of Pinhais.

Keywords: probability density function, water components, adjustment.

\section{INTRODUÇÃO}

As componentes climáticas mais importantes para a elaboração de projetos agrícolas e manejo da irrigação são aquelas que afetam a quantidade de água que será ou poderá ser aproveitada pela planta, sendo que a P e ETo são reconhecidas, normalmente, como as variáveis mais importantes (BASTOS et al., 1994; RIBEIRO; LUNARDI, 1997; ALBUQUERQUE et al., 2010). Assim, o conhecimento da $\mathrm{P}$ e ETo prováveis, a partir do ajustamento e obtenção de parâmetros estatísticos referentes a essas variáveis é útil e aplicável à atividade agrícola como um todo (ÁVILA et al., 2009; SOUZA et al. 2017).

$\mathrm{O}$ estudo de diferentes níveis de probabilidade de um evento meteorológico, como a ETo possibilita a quantificação da vazão necessária para irrigar determinada área, a partir da escolha de valores representativos e confiáveis, considerando o retorno econômico e social preconizado para o projeto (BASTOS et al., 1994). Portanto, a disponibilidade de séries históricas consistentes de $\mathrm{P}$ e ETo é indispensável para a determinação do padrão pluviométrico e evapotranspirométrico de uma região (SEDIYAMA et al., 1996; JERSZURKI et al. 2012; JERSZURKI et al. 2015).

A quantidade mínima de precipitação pluviométrica esperada à determinada probabilidade de ocorrência é denominada precipitação provável e pode ser estimada ajustando-se a série histórica de dados de precipitação a uma função densidade de probabilidade (FDP). A evapotranspiração provável é a lâmina máxima estimada com uma FDP, que pode ser igualada ou superada a determinado nível de probabilidade (FRIZZONE et al., 2005; SOUZA et al. 2017). Existem diversas FDP, não havendo uma específica que justifique o seu uso generalizado no estudo de variáveis ambientais (BACK, 2001). Assim, as FDP são selecionadas conforme o critério de melhor ajuste com séries históricas, facilidade de estimativa de seus parâmetros e flexibilidade computacional.

Segundo Lanna (2001), Sampaio et al. (2007) e Amburn et al. (2015) as FDP Gama e Log-Normal são as mais utilizadas para fazer a estimativa da precipitação. As FDP Normal, Beta e Gumbel são as mais usadas para estimar a evapotranspiração (PRUITT et al., 1972). Gurski (2018) comenta que embora a FDP Gama seja de uso consagrado na determinação da $\mathrm{P}$ provável para períodos inferiores a um mês, outras FDP devem ser consideradas para estimá-la, pois a Gama não apresenta o melhor ajuste em todos os decêndios analisados.

Quanto à estimativa dos valores prováveis a partir da FDP que melhor se ajustou à série de dados estudada, Saad (1990) comenta que em condições de irrigação tipicamente suplementar, dificilmente a 
economia dos projetos de irrigação justifica a escolha de níveis de probabilidade superior a $90 \%$. Na prática, os valores usuais adotados variam entre $50 \%$ e $75 \%$, dependendo das implicações econômicas associadas ao projeto em consideração.

Nesse contexto, teve-se por objetivo no presente trabalho determinar os parâmetros estatísticos da função densidade de probabilidade (FDP) com melhor ajuste aos valores decendiais de precipitação pluvial (P) e evapotranspiração de referência (ETo) para o município de Pinhais-PR, e calcular os valores decendiais de precipitação a $75 \%$ de probabilidade e ETo a $25 \%$.

$$
\begin{array}{cr}
\mathrm{ETo}_{\mathrm{Ti}}=\frac{\mathrm{N}_{\mathrm{i}}}{12} \cdot \frac{1}{30} \cdot 16 \cdot\left(\frac{10 \cdot \mathrm{T}_{\text {med }}}{\mathrm{I}}\right)^{\mathrm{a}}, & \text { sendo: } \mathrm{T}_{\text {med } \mathrm{i}}>0{ }^{\circ} \mathrm{C} \\
a=6,75 \cdot 10^{-7} \cdot I^{3}-7,71 \cdot 10^{-5} \cdot I^{2}+1,7912 \cdot 10^{-2} \cdot I+0,49239, \\
a=\sum_{i=1}^{12}\left(0,2 \cdot T_{m}\right)^{1,514}, & \text { sendo } \mathrm{T}_{\mathrm{m}}>0{ }^{\circ} \mathrm{C}
\end{array}
$$

em que: $\mathrm{ETo}_{\mathrm{Ti}}$ - evapotranspiração de referência do i-ésimo dia $\left(\mathrm{mm} \mathrm{dia}{ }^{-1}\right) ; \mathrm{N}_{\mathrm{i}}-$ fotoperíodo no i-ésimo dia (horas); $\mathrm{T}_{\text {med } \mathrm{i}}-$ temperatura média do ar no i-ésimo dia $\left({ }^{\circ} \mathrm{C}\right)$; a - função cúbica do índice de calor I da região (adimensional); I - índice de calor da região (adimensional); $\mathrm{T}_{\mathrm{m}}-$ temperatura média normal do m-ésimo mês do ano $\left({ }^{\circ} \mathrm{C}\right)$.

Considerando os procedimentos metodológicos de Souza et al. (2013) e Souza (2018), o cálculo da P e ETo decendial provável foram organizados e realizados com o auxílio de uma planilha eletrônica, contendo rotinas desenvolvidas especialmente para essa finalidade, realizando os seguintes passos: (a) Agrupamento dos valores diários de P e ETo em períodos de dez dias (decêndios); (b) Estabelecimento das distribuições de frequência com os dados das séries observadas; (c) Cálculo dos parâmetros estatísticos das funções densidade de probabilidade Exponencial, Gama, Normal, Triangular e Uniforme, com as séries de valores decendiais; (d) Verificação da aderência dos valores decendiais às cinco FDP com o teste de Kolmogorov-Smirnov a 5\% de significância; (e) Escolha da FDP que melhor se ajustou a cada decêndio observado; (f) Determinação dos valores prováveis com diferentes níveis de probabilidade de ocorrência; (g) Os valores diários P e ETo foram organizados em 37 decêndios por ano. O trigésimo sétimo decêndio de cada ano foi composto pelos cinco ou seis (ano bissexto) últimos dias do ano. No caso da $\mathrm{P}$, os dados diários foram tabulados e agrupados, separando os decêndios com valor igual à zero dos diferentes de zero. Para evitar inconsistências na estimativa dos parâmetros das FDP utilizadas, os decêndios com valor inferior a $1,0 \mathrm{~mm}$ foram considerados iguais a zero.

Foram consideradas as FDP Gama, Normal, Exponencial, Triangular e Uniforme. Os parâmetros estatísticos determinados foram: alfa e beta, para a FDP Gama; média e desvio padrão, para a Normal; maior valor, menor valor e moda, para a Triangular; média, 
para a Exponencial; e maior e menor valor, para a Uniforme (ASSIS et al., 1996; SOUZA, 2018). A aderência dos valores decendiais às FDP foi obtida por meio do teste de Kolmogorov-Smirnov a 5\% de significância.

$$
F(p)=P_{0}+\left(1-P_{0}\right) \cdot D(p),
$$

em que: $\mathrm{F}(\mathrm{p})$ - função cumulativa de probabilidade da distribuição mista $(\%) ; \mathrm{P}_{\mathrm{o}}-$ probabilidade de ocorrência de decêndios com valor igual a zero $(\%)$ ou com valores menores que $1 \mathrm{~mm} ; \mathrm{D}(\mathrm{p})$ - probabilidade estimada com a FDP cumulativa teórica de melhor ajuste, cujos parâmetros foram determinados na ausência de decêndios com valor igual a zero $(\%)$.

$$
\begin{gathered}
F P_{75 \%}=P .\left(P_{75 \%} \in P: P_{i} \geq P_{75 \%}\right)=75 \% \\
E \text { ETo }_{25 \%}=P .\left(\text { ETo }_{25 \%} \in \text { ETo }: \text { ETo }_{i} \geq E T o_{25 \%}\right)=25 \%
\end{gathered}
$$

Logo, para cada decêndio, a $\mathrm{P}_{75 \%}$ referese ao valor de $\mathrm{P}_{\mathrm{i}}$ que tenha $75 \%$ de probabilidade de ser igualado ou superado, o que corresponde à probabilidade da precipitação $\mathrm{P}_{\mathrm{i}}$ ocorrer três vezes a cada quatro anos ou com tempo de retorno $\mathrm{T}=1,33$ anos, em média. Para cada decêndio, a $\mathrm{ETo}_{25 \%}$ refere-se ao valor de evapotranspiração $\mathrm{ETo}_{\mathrm{i}}$ que tenha $25 \%$ de probabilidade de ser igualado ou superado, o que corresponde à probabilidade da evapotranspiração $\mathrm{ETo}_{\mathrm{i}}$ ocorrer uma vez a cada quatro anos ou com tempo de retorno $\mathrm{T}=4$ anos, em média.

\section{RESULTADOS E DISCUSSÃO}

Os resultados apresentados na Tabela 1 permitem verificar que as FDP que melhor se ajustaram aos dados de ETo foram a Normal e Gama. Os valores decendiais da ETo tiveram tendência de se distribuírem normalmente
$(51,4 \%)$. No entanto, mediante os resultados obtidos para as cinco FDP é importante observar que não se deve assumir sempre que os valores decendiais de ETo seguem uma FDP Normal, pois para a localidade estudada, aproximadamente, metade dos decêndios ajustaram-se a outras FDP.

Analisando-se a diferença (amplitudes) entre os valores de ETo média e $\mathrm{ETO}_{25 \%}$ (Figura 1) ao longo dos decêndios do ano, verificou-se que a média das amplitudes dos valores de ETo ao longo do ano foi igual a 2,1 $\mathrm{mm}$ decêndio $^{-1}\left(\mathrm{~s}=0,44 \mathrm{~mm}\right.$ decêndio $\left.{ }^{-1}\right)$. Percentualmente, a média das amplitudes ficou em 11,1\% (s = 3,7\%). Os meses de junho e julho apresentaram as maiores amplitudes percentuais, sendo iguais a $15,6 \%$ e $17,1 \%$, respectivamente. O mês de fevereiro apresentou a menor amplitude percentual, sendo iguais $5,7 \%$. 


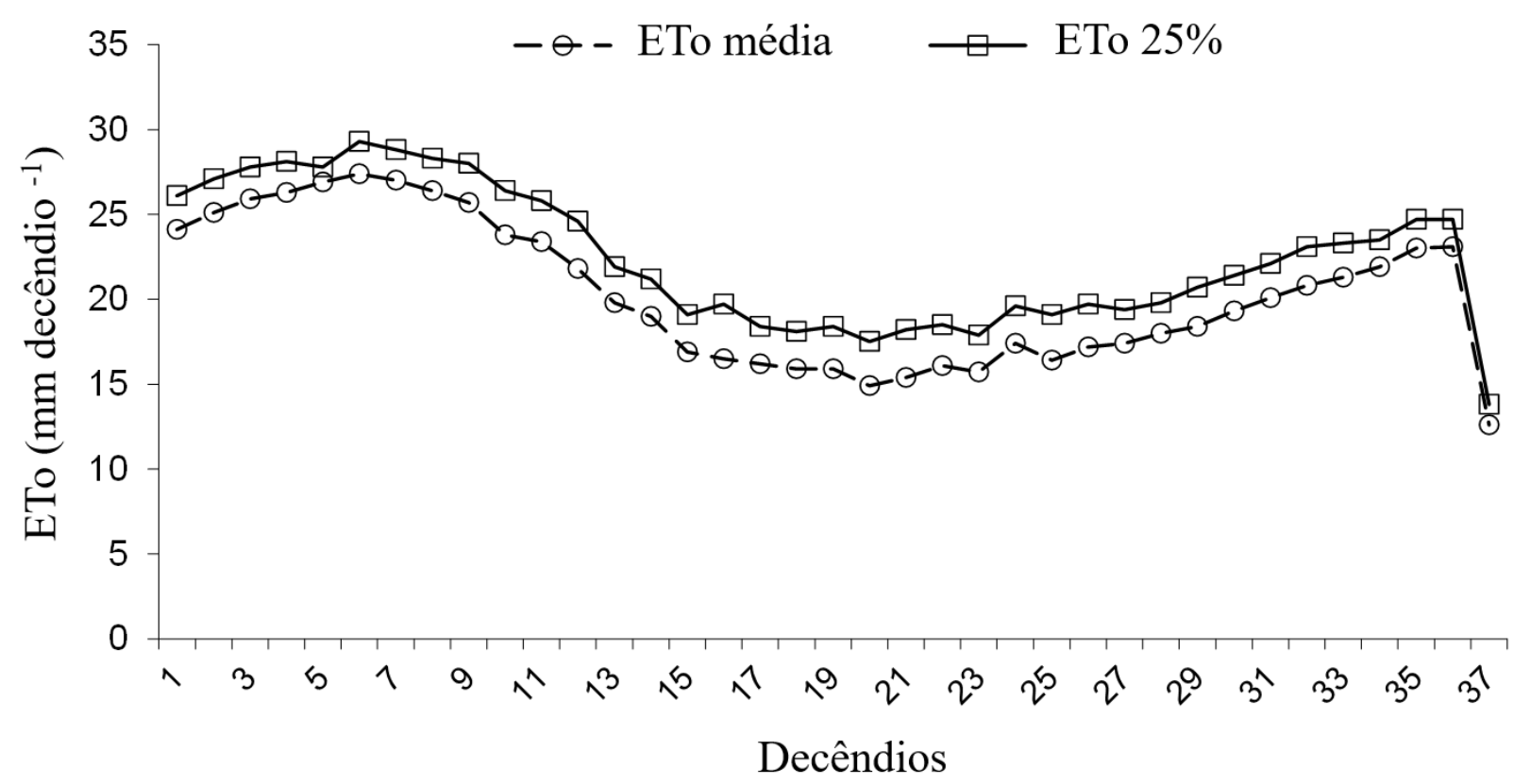

Figura 1. Evapotranspiração de referência (ETo) média e a $25 \%$ probabilidade $\left(\mathrm{ETo}_{25 \%}\right)$, calculados com a função densidade de probabilidade de melhor ajuste, para os 37 decêndios do ano, para o município de Pinhais - PR.

Tabela 1. Parâmetros estatísticos de cinco funções densidade de probabilidade - FDP (N - Normal, T - Triangular, G - Gama, E - Exponencial e U - Uniforme) e valores prováveis decendiais da ETo $\left(\mathrm{ETo}_{25 \%}\right)$, para os 37 decêndios do ano, considerando um período de 33 anos, no município de Pinhais - PR

\begin{tabular}{|c|c|c|c|c|c|c|c|c|c|}
\hline \multicolumn{2}{|c|}{ 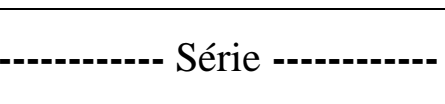 } & \multicolumn{7}{|c|}{ 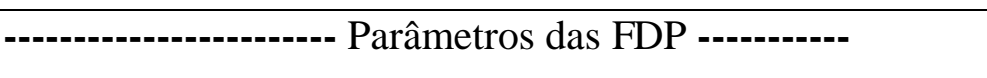 } & \multirow{2}{*}{$\begin{array}{c}\mathrm{ETo}_{25 \%} * * * \\
(\mathrm{~mm} \\
\left.\text { decêndio }^{-1}\right)\end{array}$} \\
\hline Decêndios & Anos & $\begin{array}{l}\text { Menor } \\
\text { Valor }\end{array}$ & $\begin{array}{l}\text { Maior } \\
\text { Valor }\end{array}$ & $\mu^{*}$ & $\sigma^{*}$ & Alfa & Beta & FDP** & \\
\hline $1(01 / 01$ a $10 / 01)$ & 34 & 19,5 & 30,1 & 24,1 & 2,9 & 71,42 & 0,34 & $\mathrm{~T}$ & 26,1 \\
\hline $2(11 / 01$ a $20 / 01)$ & 34 & 18,3 & 32,3 & 25,1 & 3,0 & 72,52 & 0,35 & $\mathrm{~N}$ & 27,1 \\
\hline $3(21 / 01$ a 30/01) & 32 & 20,4 & 31,4 & 25,9 & 2,9 & 80,74 & 0,32 & $\mathrm{~N}$ & 27,8 \\
\hline $4(31 / 02$ a 09/02) & 34 & 20,3 & 31,8 & 26,3 & 2,6 & 100,37 & 0,26 & $\mathrm{~N}$ & 28,1 \\
\hline $5(10 / 02$ a 19/02) & 34 & 21,4 & 30,8 & 26,9 & 2,6 & 104,28 & 0,26 & $\mathrm{~T}$ & 27,8 \\
\hline $6(20 / 02$ a 01/03) & 34 & 21,0 & 32,7 & 27,4 & 2,9 & 91,05 & 0,30 & $\mathrm{G}$ & 29,3 \\
\hline $7(02 / 03$ a 11/03) & 34 & 22,0 & 31,8 & 27,0 & 2,6 & 112,38 & 0,24 & $\mathrm{~N}$ & 28,8 \\
\hline $8(12 / 03$ a $21 / 03)$ & 34 & 21,4 & 34,1 & 26,4 & 2,7 & 99,52 & 0,27 & $\mathrm{~N}$ & 28,3 \\
\hline $9(22 / 03$ a $31 / 03)$ & 34 & 19,7 & 33,3 & 25,7 & 3,4 & 61,91 & 0,42 & $\mathrm{~N}$ & 28,0 \\
\hline $10(01 / 04$ a $10 / 04)$ & 34 & 17,0 & 31,1 & 23,8 & 3,8 & 40,70 & 0,59 & $\mathrm{~N}$ & 26,4 \\
\hline $11(11 / 04$ a 20/04) & 34 & 17,9 & 31,4 & 23,4 & 3,7 & 42,17 & 0,56 & G & 25,8 \\
\hline $12(21 / 04$ a $30 / 04)$ & 34 & 13,9 & 31,2 & 21,8 & 4,1 & 28,54 & 0,76 & $\mathrm{~N}$ & 24,6 \\
\hline $13(01 / 05$ a $10 / 05)$ & 34 & 13,9 & 26,2 & 19,8 & 3,3 & 37,86 & 0,52 & $\mathrm{G}$ & 21,9 \\
\hline $14(11 / 05$ a $20 / 05)$ & 34 & 11,0 & 26,3 & 19,0 & 3,5 & 29,89 & 0,64 & $\mathrm{G}$ & 21,2 \\
\hline $15(21 / 05$ a $30 / 05)$ & 34 & 10,0 & 25,5 & 16,9 & 3,3 & 26,07 & 0,65 & $\mathrm{~N}$ & 19,1 \\
\hline $16(31 / 05$ a 09/06) & 34 & 9,0 & 26,2 & 16,5 & 4,8 & 11,97 & 1,38 & $\mathrm{~N}$ & 19,7 \\
\hline $17(10 / 06$ a 19/06) & 34 & 8,2 & 23,8 & 16,2 & 3,6 & 20,06 & 0,81 & $\mathrm{G}$ & 18,4 \\
\hline $18(20 / 06$ a $29 / 06)$ & 34 & 9,7 & 23,5 & 15,9 & 3,3 & 22,08 & 0,72 & $\mathrm{~N}$ & 18,1 \\
\hline
\end{tabular}


Souza et al.

\begin{tabular}{lccccccccc}
\hline $19(30 / 06$ a 09/07) & 34 & 8,9 & 21,6 & 15,9 & 3,7 & 17,49 & 0,91 & $\mathrm{~N}$ & 18,4 \\
$20(10 / 07$ a $19 / 07)$ & 34 & 7,5 & 27,4 & 14,9 & 4,4 & 12,54 & 1,19 & $\mathrm{G}$ & 17,5 \\
$21(20 / 07$ a $29 / 07)$ & 34 & 7,7 & 21,7 & 15,4 & 4,1 & 13,08 & 1,18 & $\mathrm{~N}$ & 18,2 \\
$22(30 / 07$ a 08/08) & 34 & 9,5 & 23,5 & 16,1 & 3,8 & 18,64 & 0,86 & $\mathrm{G}$ & 18,5 \\
$23(09 / 08$ a 18/08) & 34 & 10,3 & 23,3 & 15,7 & 3,5 & 21,16 & 0,74 & $\mathrm{G}$ & 17,9 \\
$24(19 / 08$ a 28/08) & 34 & 10,2 & 23,7 & 17,4 & 3,3 & 26,52 & 0,65 & $\mathrm{~N}$ & 19,6 \\
$25(29 / 08$ a 07/09) & 34 & 9,0 & 28,9 & 16,4 & 4,6 & 14,54 & 1,13 & $\mathrm{G}$ & 19,1 \\
$26(08 / 09$ a 17/09) & 34 & 9,6 & 24,8 & 17,2 & 3,6 & 22,63 & 0,76 & $\mathrm{~N}$ & 19,7 \\
$27(18 / 09$ a 27/09) & 34 & 11,2 & 27,3 & 17,4 & 3,3 & 31,28 & 0,56 & $\mathrm{G}$ & 19,4 \\
$28(28 / 09$ a 07/10) & 34 & 14,0 & 26,3 & 18,0 & 3,0 & 40,02 & 0,45 & $\mathrm{G}$ & 19,8 \\
$29(08 / 10$ a $17 / 10)$ & 34 & 11,6 & 29,7 & 18,4 & 3,8 & 25,73 & 0,71 & $\mathrm{G}$ & 20,7 \\
$30(18 / 10$ a 27/10) & 34 & 13,5 & 26,7 & 19,3 & 3,1 & 40,92 & 0,47 & $\mathrm{~N}$ & 21,4 \\
$31(28 / 10$ a 06/11) & 34 & 15,2 & 27,3 & 20,1 & 3,0 & 47,01 & 0,43 & $\mathrm{~N}$ & 22,1 \\
$32(07 / 11$ a 16/11) & 34 & 14,2 & 28,0 & 20,8 & 3,5 & 35,60 & 0,59 & $\mathrm{G}$ & 23,1 \\
$33(17 / 11$ a 26/11) & 34 & 13,9 & 27,1 & 21,3 & 3,1 & 45,93 & 0,46 & $\mathrm{G}$ & 23,3 \\
$34(27 / 11$ a 06/12) & 34 & 17,5 & 26,5 & 21,9 & 2,4 & 84,77 & 0,26 & $\mathrm{~N}$ & 23,5 \\
$35(07 / 12$ a 16/12) & 34 & 17,3 & 27,2 & 23,0 & 2,6 & 76,66 & 0,30 & $\mathrm{~N}$ & 24,7 \\
$36(17 / 12$ a 26/12) & 34 & 19,1 & 28,6 & 23,1 & 2,5 & 90,80 & 0,25 & $\mathrm{G}$ & 24,7 \\
$37(27 / 12$ a 31/12) & 34 & 9,1 & 17,7 & 12,6 & 2,0 & 40,88 & 0,31 & $\mathrm{G}$ & 13,8 \\
\hline
\end{tabular}

* média $(\mu)$ e desvio padrão $(\sigma)$ dos valores decendias da série.

** função densidade de probabilidade que melhor se ajustou aos dados decendiais da série.

*** evapotranspiração de referência a $25 \%$ de probabilidade.

Analisando-se os ajustamentos das funções densidade de probabilidade (FDP) aos dados de precipitação e valores de precipitação provável estimados a $75 \%$ de probabilidade (Tabela 2), verificou-se que as FDP Normal, Gama e Exponencial, ajustaramse bem aos dados de precipitação de 7 $(18,9 \%), 20(54,1 \%)$ e $10(27,0 \%)$ decêndios, respectivamente. As FDP Triangular e Uniforme não foram melhores em nenhum ajustamento. De forma geral, os valores decendiais da precipitação tenderam para a FDP Gama. No entanto, mediante os resultados obtidos para as cinco FDP é importante observar novamente, assim como foi comentado para ETo, que não se deve assumir sempre que os valores decendiais de precipitação seguem a uma FDP Gama, pois aproximadamente metade dos decêndios ajustaram-se a outras FDP. Gurski (2018), estudando componentes hídricas no estado do Paraná, também verificou que a distribuição Gama apresentou melhor ajuste em aproximadamente $59,8 \%$ dos decêndios de precipitação analisados. Logo, 40,2\% foram melhor ajustadas em outras FDP. Muitos estudos demonstraram que a FDP Gama é a que melhor se aplica aos estudos envolvendo chuva provável (PAULO et al., 2016; ALVARENGA et al., 2018). A FDP Gama possui bom ajuste para variáveis contínuas que tenham limite inferior igual a zero e não possuam limite superior, sendo por isso largamente utilizada para o estudo de séries históricas de precipitação (SOUZA, 2018). No entanto, Souza et al. (2013), Jerszurki et al. $(2012,2015)$ verificaram a aderência de outras FDP, principalmente quando a série histórica de dados diários de chuva (extensa ou curta) apresenta poucos registros com precipitação superior a $1,0 \mathrm{~mm}$ no período, como ocorre em períodos secos e veranicos. 
Tabela 2. Parâmetros estatísticos de cinco funções densidade de probabilidade ( $\mathrm{N}$ - Normal, $\mathrm{T}$ Triangular, G - Gama, E - Exponencial e U - Uniforme) e valores prováveis decendiais da $\mathrm{P}\left(\mathrm{P}_{75 \%}\right)$, para os 37 decêndios do ano, considerando um período de 33 anos, no município de Pinhais - PR

\begin{tabular}{|c|c|c|c|c|c|c|c|c|c|c|}
\hline \multicolumn{2}{|c|}{----------- Série --------- } & \multicolumn{6}{|c|}{------------------- Parâmetros das FDP ------ } & \multicolumn{2}{|c|}{$\mathrm{P}(\mathrm{P}=0)$} & \multirow{2}{*}{$\begin{array}{c}\mathrm{P}_{75 \%} \\
\left(\mathrm{~mm} \mathrm{decêndio}^{-1}\right)\end{array}$} \\
\hline Decêndios & Anos & $\begin{array}{c}\text { Menor } \\
\text { valor }\end{array}$ & $\begin{array}{c}\text { Maior } \\
\text { valor }\end{array}$ & $\mu^{*}$ & $\sigma^{*}$ & Alfa & Beta & $\mathrm{FDP}^{* *}$ & $(\%)$ & \\
\hline $1(01 / 01$ a $10 / 01)$ & 32 & 5,6 & 251,6 & 62,1 & 57,0 & 1,40 & 44,22 & $\mathrm{G}$ & 2,9 & 22,0 \\
\hline $2(11 / 01$ a $20 / 01)$ & 34 & 5,7 & 200,1 & 59,0 & 42,0 & 2,06 & 28,67 & $\mathrm{G}$ & 0,0 & 28,8 \\
\hline $3(21 / 01$ a $30 / 01)$ & 31 & 2,8 & 204,6 & 68,8 & 43,8 & 1,80 & 38,16 & $\mathrm{~N}$ & 5,9 & 32,5 \\
\hline $4(31 / 02$ a $09 / 02)$ & 32 & 3,0 & 137,8 & 59,8 & 38,9 & 2,02 & 29,63 & G & 5,7 & 25,2 \\
\hline $5(10 / 02$ a $19 / 02)$ & 33 & 5,9 & 138,5 & 56,0 & 39,3 & 1,67 & 33,52 & $\mathrm{~N}$ & 2,9 & 26,7 \\
\hline $6(20 / 02$ a 01/03) & 33 & 1,9 & 145,9 & 48,2 & 34,8 & 1,60 & 30,17 & $\mathrm{G}$ & 2,9 & 18,8 \\
\hline $7(02 / 03$ a 11/03) & 33 & 4,7 & 124,6 & 45,1 & 33,8 & 1,96 & 23,04 & $\mathrm{G}$ & 0,0 & 21,4 \\
\hline $8(12 / 03$ a $21 / 03)$ & 33 & 1,1 & 110,2 & 37,6 & 32,1 & 1,27 & 29,71 & $\mathrm{E}$ & 2,9 & 9,7 \\
\hline $9(22 / 03$ a $31 / 03)$ & 32 & 1,3 & 174,6 & 40,0 & 34,8 & 1,31 & 30,59 & $\mathrm{G}$ & 5,7 & 12,2 \\
\hline $10(01 / 04$ a $10 / 04)$ & 32 & 1,5 & 96,9 & 27,0 & 24,8 & 1,20 & 22,58 & $\mathrm{E}$ & 5,7 & 6,2 \\
\hline $11(11 / 04$ a $20 / 04)$ & 32 & 3,0 & 131,6 & 34,9 & 27,8 & 1,56 & 22,32 & $\mathrm{G}$ & 5,7 & 12,3 \\
\hline $12(21 / 04$ a $30 / 04)$ & 29 & 1,0 & 87,5 & 21,3 & 21,9 & 0,98 & 21,73 & $\mathrm{E}$ & 14,3 & 2,8 \\
\hline $13(01 / 05$ a $10 / 05)$ & 27 & 1,4 & 114,8 & 36,4 & 35,2 & 0,92 & 39,73 & G & 20,0 & 1,9 \\
\hline $14(11 / 05$ a $20 / 05)$ & 31 & 1,0 & 136,5 & 37,6 & 38,5 & 0,83 & 45,60 & G & 8,6 & 5,6 \\
\hline $15(21 / 05$ a $30 / 05)$ & 29 & 2,0 & 121,3 & 32,7 & 33,8 & 1,11 & 29,42 & $\mathrm{E}$ & 14,3 & 4,4 \\
\hline $16(31 / 05$ a $09 / 06)$ & 33 & 1,0 & 88,2 & 30,9 & 26,6 & 1,05 & 29,38 & $\mathrm{E}$ & 2,9 & 8,0 \\
\hline $17(10 / 06$ a $19 / 06)$ & 29 & 1,6 & 105,9 & 33,2 & 27,8 & 1,29 & 25,81 & $\mathrm{G}$ & 14,3 & 6,4 \\
\hline $18(20 / 06$ a $29 / 06)$ & 28 & 2,4 & 186,2 & 45,8 & 43,9 & 1,00 & 45,63 & $\mathrm{E}$ & 17,1 & 4,6 \\
\hline $19(30 / 06$ a 09/07) & 28 & 5,8 & 150,4 & 48,9 & 39,3 & 1,76 & 27,84 & $\mathrm{G}$ & 17,1 & 11,0 \\
\hline $20(10 / 07$ a $19 / 07)$ & 25 & 1,7 & 98,8 & 31,2 & 31,3 & 0,93 & 33,49 & $\mathrm{G}$ & 25,7 & 0,0 \\
\hline $21(20 / 07$ a 29/07) & 26 & 1,3 & 123,0 & 35,9 & 34,6 & 1,10 & 32,74 & $\mathrm{E}$ & 20,0 & 2,3 \\
\hline $22(30 / 07$ a $08 / 08)$ & 24 & 1,0 & 93,2 & 30,0 & 26,7 & 0,91 & 33,07 & $\mathrm{E}$ & 28,6 & 0,0 \\
\hline $23(09 / 08$ a $18 / 08)$ & 26 & 1,0 & 154,6 & 32,3 & 36,4 & 0,76 & 42,35 & $\mathrm{E}$ & 22,9 & 1,0 \\
\hline $24(19 / 08$ a $28 / 08)$ & 24 & 1,0 & 122,9 & 34,0 & 33,0 & 0,97 & 34,95 & $\mathrm{E}$ & 28,6 & 0,0 \\
\hline $25(29 / 08$ a $07 / 09)$ & 32 & 1,3 & 134,7 & 35,3 & 30,9 & 1,14 & 30,92 & $\mathrm{G}$ & 5,7 & 9,4 \\
\hline $26(08 / 09$ a $17 / 09)$ & 28 & 1,8 & 114,8 & 46,6 & 31,2 & 1,55 & 30,10 & $\mathrm{~N}$ & 17,1 & 5,6 \\
\hline $27(18 / 09$ a $27 / 09)$ & 31 & 1,6 & 152,8 & 45,4 & 37,0 & 1,57 & 28,94 & $\mathrm{G}$ & 8,6 & 14,5 \\
\hline $28(28 / 09$ a $07 / 10)$ & 33 & 2,3 & 162,1 & 49,1 & 40,9 & 1,26 & 38,97 & $\mathrm{G}$ & 2,9 & 15,9 \\
\hline $29(08 / 10$ a $17 / 10)$ & 33 & 2,0 & 85,0 & 34,9 & 22,0 & 2,07 & 16,83 & G & 2,9 & 16,1 \\
\hline $30(18 / 10$ a $27 / 10)$ & 34 & 2,2 & 102,7 & 45,7 & 27,8 & 1,86 & 24,55 & $\mathrm{~N}$ & 0,0 & 27,0 \\
\hline $31(28 / 10$ a $06 / 11)$ & 33 & 1,3 & 100,1 & 37,4 & 24,5 & 1,48 & 25,30 & $\mathrm{~N}$ & 2,9 & 19,2 \\
\hline $32(07 / 11$ a $16 / 11)$ & 31 & 1,0 & 128,2 & 43,3 & 27,2 & 1,87 & 23,16 & $\mathrm{~N}$ & 8,6 & 18,3 \\
\hline $33(17 / 11$ a $26 / 11)$ & 31 & 3,7 & 99,5 & 38,7 & 28,6 & 1,57 & 24,63 & G & 8,6 & 12,4 \\
\hline $34(27 / 11$ a $06 / 12)$ & 33 & 1,2 & 127,0 & 40,8 & 29,8 & 1,73 & 23,58 & $\mathrm{~N}$ & 0,0 & 20,7 \\
\hline $35(07 / 12$ a $16 / 12)$ & 33 & 7,2 & 161,1 & 44,7 & 34,5 & 1,72 & 25,92 & $\mathrm{G}$ & 2,9 & 18,4 \\
\hline $36(17 / 12$ a $26 / 12)$ & 34 & 2,1 & 201,1 & 52,0 & 41,0 & 1,65 & 31,59 & $\mathrm{G}$ & 0,0 & 22,3 \\
\hline $37(27 / 12$ a $31 / 12)$ & 32 & 1,0 & 120,6 & 28,6 & 30,6 & 0,88 & 32,39 & G & 5,7 & 5,6 \\
\hline
\end{tabular}

* média $(\mu)$ e desvio padrão $(\sigma)$ dos valores decendiais da série.

** função densidade de probabilidade que melhor se ajustou aos dados decendiais da série.

Analisando-se a diferença (amplitudes) entre os valores de precipitação média e $\mathrm{P}_{75 \%}$ (Figura 2) ao longo dos decêndios do ano, verificou-se que a média das amplitudes dos valores de precipitação ao longo do ano foi igual a $28,7 \mathrm{~mm}$ decêndio $^{-1}(\mathrm{~s}=6,4 \mathrm{~mm}$ 
decêndio $^{-1}$ ). Percentualmente, a média das amplitudes ficou em $71,8 \%$. Os meses de janeiro e julho apresentam as maiores amplitudes, sendo iguais a 35,5 e $34,2 \mathrm{~mm}$ decêndio $^{-1}$, respectivamente. Os meses de abril, outubro, novembro e dezembro apresentam as menores amplitudes, sendo iguais a 20,6;23,6;23,2 e 24,8 mm decêndio $^{-1}$, respectivamente.

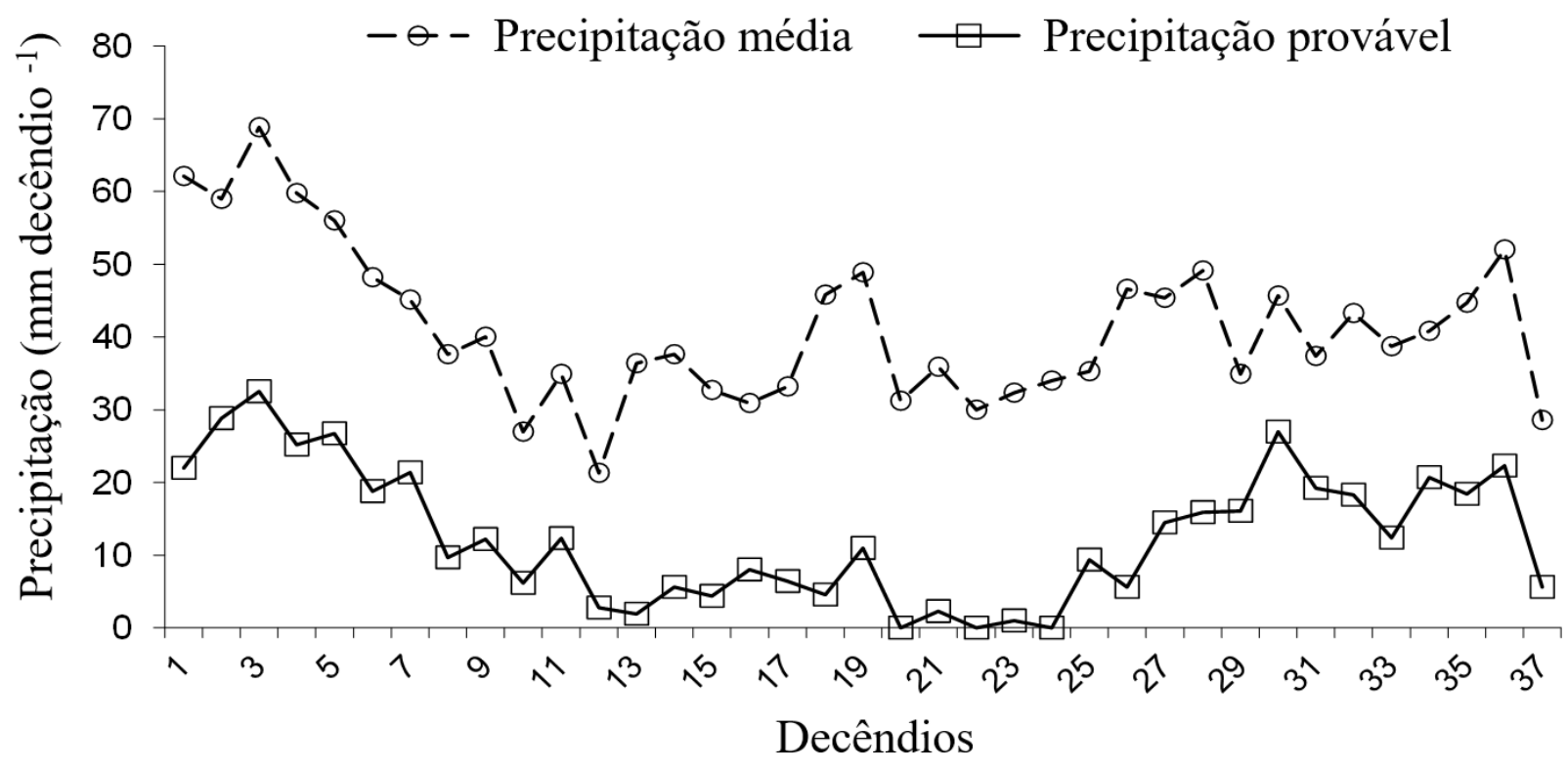

Figura 2. Precipitação média e provável a $75 \%$ de probabilidade $\left(\mathrm{P}_{75 \%}\right)$, calculados com a função densidade de probabilidade de melhor ajuste, para os 37 decêndios do ano, para o município de Pinhais - PR.

Analisando-se a diferença (amplitudes) entre os valores de $\mathrm{ETo}_{25 \%}$ e $\mathrm{P}_{75 \%}$ (Figura 3) ao longo dos decêndios do ano, verificou-se que a média das amplitudes ao longo do ano foi igual a 9,8 mm decêndio ${ }^{-1}$ ( $\mathrm{s}=7,3 \mathrm{~mm}$ decêndio $\left.{ }^{-1}\right)$. Percentualmente, a média das amplitudes ficou em 48,3\% ( $\mathrm{s}=34,9 \%$ ). Nos meses de março, abril, maio, julho e agosto teve-se maior possibilidade da ocorrência de deficiência hídrica às plantas. $\mathrm{O}$ decêndio 12 (21/04 a 30/04) apresentou a maior amplitude $\left(21,8 \mathrm{~mm}\right.$ decêndio $\left.^{-1}\right)$, indicando que o período deve ser analisado com maior cuidado pelos projetistas para dimensionar os sistemas de irrigação. As menores amplitudes ocorreram nos decêndios 2 (11/01 a 20/01), 3 $(21 / 01$ a $30 / 01)$ e $30(18 / 10$ a $27 / 10)$ e foram negativas, ou seja, a precipitação provável foi maior do que a ETo provável. Desta forma, a $75 \%$ de probabilidade, o período não deverá sofrer problemas de deficiência hídrica, mas dependendo do tipo de solo, poderá promover sua saturação. Os meses de abril e agosto apresentaram as maiores amplitudes, sendo iguais a 18,5 e $18,3 \mathrm{~mm}$ decêndio $^{-1}$, respectivamente. Os meses de janeiro e outubro apresentaram as menores amplitudes, sendo iguais a $-0,8$ e $1,0 \mathrm{~mm}$ decêndio ${ }^{-1}$, respectivamente. 


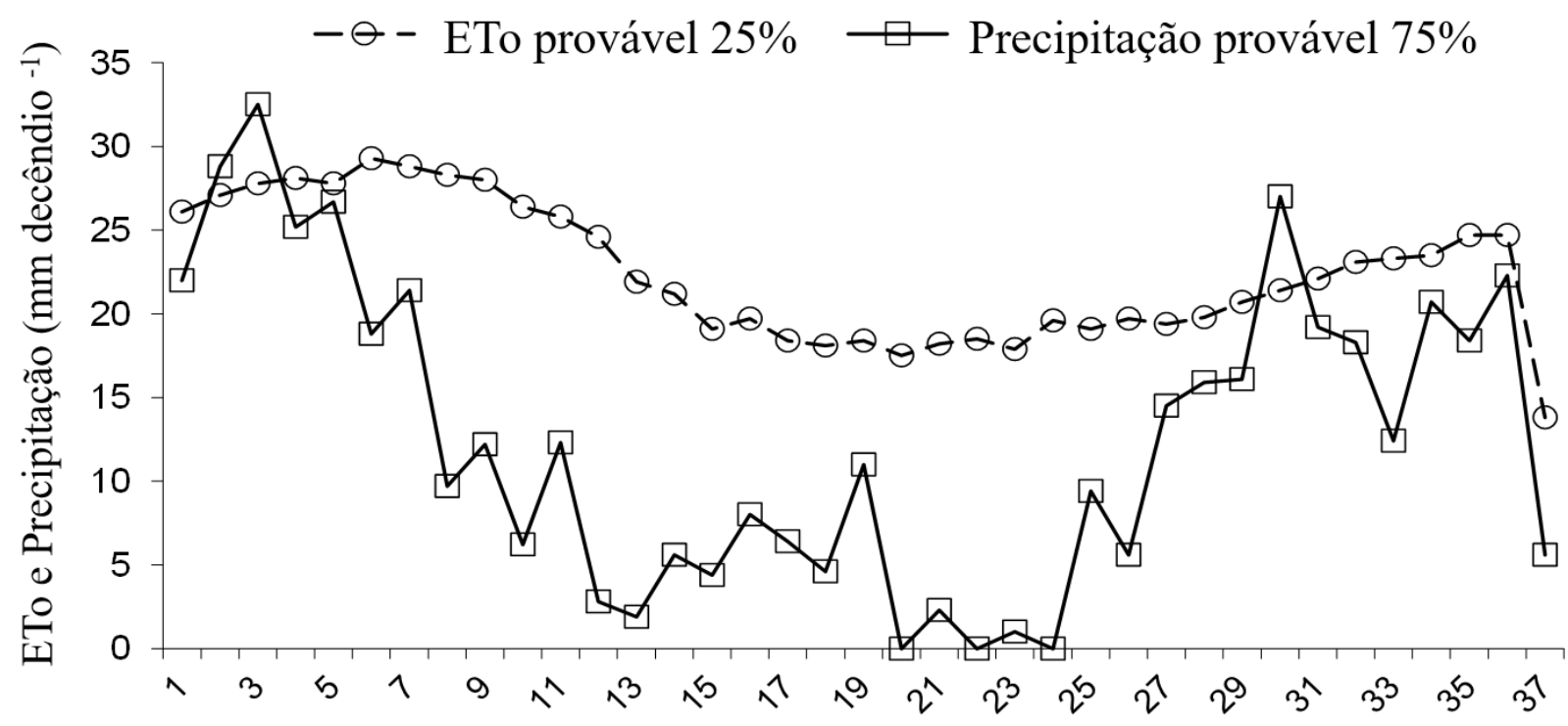

Decêndios

Figura 3. Evapotranspiração provável $\left(\mathrm{ETo}_{25 \%}\right)$ e precipitação provável $\left(\mathrm{P}_{75 \%}\right)$, calculadas com a FDP de melhor ajuste, para os 37 decêndios do ano, para o município de Pinhais.

É importante observar que as considerações feitas foram realizadas para as amplitudes entre a $\mathrm{P}_{75 \%}$ e $\mathrm{ETo}_{25 \%}$. $\mathrm{Na}$ realização de projetos de irrigação é sempre mais consistente analisar as amplitudes considerando a evapotranspiração da cultura (ETc), sendo necessário, evidentemente, o conhecimento prévio da área e das prováveis culturas que se pretende irrigar ao longo do ano.

Na Figura 4 encontram-se os diagramas de dispersão das associações entre ETo média vs ETo $_{25 \%}$ (a); e, $\mathrm{P}_{\text {média }}$ vs $\mathrm{P}_{75 \%}$ (b). Nas análises

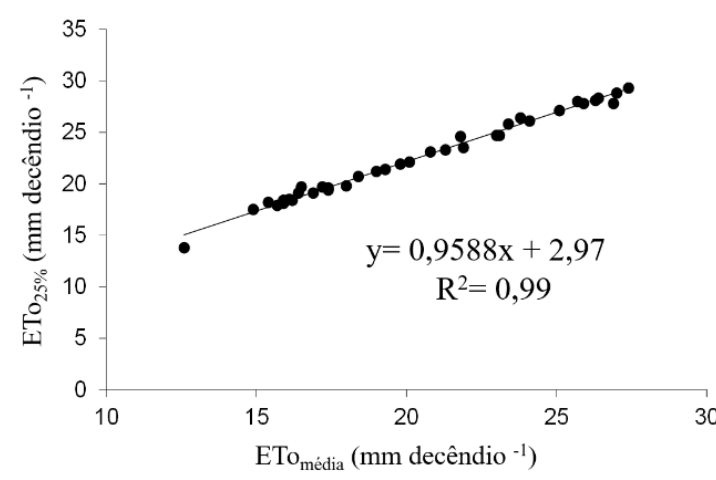

a)

Figura 4. Análise de regressão linear e respectivo coeficiente de de
médios e prováveis de P e ETo para região de Pinhais: a) ETo média vs ETo
Rev. Bras. Agric. Irr. V. 13, m $^{\circ}$ 4, Fortaleza, p. 3512 - 3523, Jul - Ago, 2019

b) de regressão e respectivos coeficientes de correlação $(\mathrm{R})$ verificou-se estreita associação entre $\mathrm{ETo}_{\text {média }}$ vs $\mathrm{ETo}_{25 \%} \quad\left(\mathrm{R}^{2}=0,99\right)$, evidenciando que valores de $\mathrm{ETo}_{25 \%}$ podem ser obtidos seguramente com valores de $\mathrm{ETo}_{\text {média }}$ decendial da região de Pinhais (Figura 4a). O ajuste dos valores de ETo à FDP Normal em $51,4 \%$ dos períodos decêndias pode ter favorecido a obtenção desse resultado, visto que a distribuição Normal tem a média e desvio padrão como parâmetros.

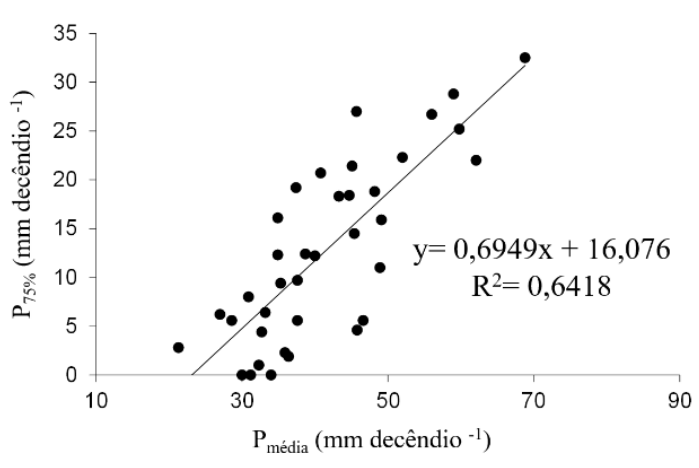

$\mathrm{P}_{\text {média }}\left(\mathrm{mm}\right.$ decêndio $\left.^{-1}\right)$ 90 
A associação entre $\mathrm{P}_{\text {média }}$ vs $\mathrm{P}_{75 \%}$ não foi tão estreita $(\mathrm{R}=0,80$; figura $4 \mathrm{~b})$ como a verificada para a ETo. Contudo, o resultado ainda é bem interessante, visto que a $\mathrm{P}$ decendial apresenta maior variabilidade que a ETo decendial. Além disso, os valores decendiais de $\mathrm{P}$ se ajustaram melhor a distribuição Gama $(54,1 \%)$, que não tem a média como parâmetro. O resultado obtido concorda com Souza et al. (2013), que ressaltaram a possibilidade de fácil obtenção da $\mathrm{P}_{75 \%}$ decendial a partir da $\mathrm{P}_{\text {média }}$ decendial, sendo importante para localidades sem estação climatológica ou contendo série histórica com falhas e poucos anos de dados. Além disso, a estimação de $\mathrm{P}_{75 \%}$ a partir de $\mathrm{P}_{\text {média }}$ pode viabilizar programas de planejamento de atividades agropecuárias mais abrangentes.

\section{CONCLUSÕES}

Apesar dos valores decendiais da evapotranspiração de referencia (ETo) terem se ajustado melhor à função densidade Normal $(51,4 \%)$ e a precipitação $(\mathrm{P})$ à função Gama (54,1\%), aproximadamente metade dos decêndios ao longo do ano ajustaram-se à outras quatro funções densidade, evidenciando a necessidade de se considerar outras funções densidade probabilidade em estudos climáticos visando a determinação de valores prováveis na região.

Os valores de $\mathrm{ETo}_{25 \%}$ e $\mathrm{P}_{75 \%}$ indicaram entre março e agosto maior possibilidade de redução no armazenamento de água no solo e ocorrência de deficiência hídrica às plantas.

Associações lineares simples possibilitam a obtenção dos valores decendiais de $\mathrm{ETo}_{25 \%}$ e $\mathrm{P}_{75 \%}$, respectivamente, com valores de $\mathrm{ETo}_{\text {médio }}$ e $\mathrm{P}_{\text {médio }}$ decendial da região de Pinhais.

\section{REFERÊNCIAS BIBLIOGRÁFICAS}

ALBUQUERQUE, M. F.; SOUZA, E. B.; OLIVEIRA. M. do C. F.; JÚNIOR, J. A. de S. Precipitação nas mesorregiões do estado do Pará: climatologia, variabilidade e tendências nas últimas décadas (1978-2008). Revista Brasileira de Climatologia, v. 6, n. 6, 2010. http://dx.doi.org/10.5380/abclima.v6i0.25606 ALVARENGA, J. S.; THEBALDI, M. S.; FERNANDES, L. R. Precipitação mensal provável do município de formiga-mg. Revista Brasileira de Agricultura Irrigada, v. $12, \quad$ n. 5, p. 2823-2833, 2018. http://dx.doi.ogr/10.7127/RBAI.V12N500714

AMBURN, S. A.; LANG, A. S.; BUONAIUTO, M. A. Precipitation forecasting with gamma distribution models for gridded precipitation events in eastern Oklahoma and northwestern Arkansas. Weather and Forecasting, v. 30, n. 2, p. 349367, 2015. DOI:10.1175/WAF-D-14-00054.1

ASSIS, F. N.; ARRUDA, H. V.; PEREIRA, A. R. Aplicações de estatística a climatologia: Teoria e prática. Pelotas: ed. Universitária/UFPel, 1996.

ÁVILA, L. F.; MELLO, C. R.; VIOLA, M. R. Mapeamento da precipitação mínima provável para o sul de Minas Gerais. Revista Brasileira de Engenharia Agrícola e Ambiental, v. 13, p. 906-915, 2009.

BACK, A. J. Seleção de distribuição de probabilidade para chuvas diárias extremas do Estado de Santa Catarina. Revista Brasileira de Meteorologia, v. 16, p. 211-222, 2001.

BASTOS, E.; AGUIAR, O. N.; ANDRADE JÚNIOR, A. S.; OLIVEIRA, C. Distribuição de frequência da evapotranspiração potencial para a região de Teresina-PI, através do modelo de Gumbel. Engenharia Agrícola, v. 14, p. 99-104, 1994.

FRIZZONE, J. A.; ANDRADE JÚNIOR, A. S.; SOUZA, J. L. M.; ZOCOLER, J. L. Planejamento de irrigação: análise e decisão de investimento. Brasília: Embrapa, 2005. $627 \mathrm{p}$.

GURSKI, B. C. Componentes hídricas prováveis e zoneamento de risco agroclimático para o Estado do Paraná. Curitiba. 2018. 134f. Tese (Doutorado em 
Agronomia, área de concentração Ciência do Solo) - Setor de Ciências Agrárias, Universidade Federal do Paraná.

JERSZURKI, D.; SOUZA, J. L. M.; EVANGELISTA, A. W. P. Distribuição de probabilidade e movimento temporal da precipitação na região de Telêmaco Borba, Paraná. Revista Brasileira de Ciências Agrárias, v. 10, n. 1, p. 110-116, 2015. DOI:10.5039/agraria.v10i1a5159.

JERSZURKI, D.; SOUZA, J. L. M.; EVANGELISTA, A. W. P. Evapotranspiração de Referência: Distribuição de probabilidade e decomposição de séries temporais para a região de Telêmaco Borba, Estado do Paraná. In: IX REUNIÃO SUL-BRASILEIRA DE CIÊNCIA DO SOLO (2012, Lages). Anais. Lages: SBCS, 2012. 4p.

LANNA, A. E. Elementos de estatística e probabilidades. In: TUCCI, C.E.M. Hidrologia: ciência e aplicação. Porto Alegre, RS: Universidade Federal do Rio Grande do Sul, 2001. p. 79-176.

PAULO, A., MARTINS, D; PEREIRA, L. S. Influence of precipitation changes on the SPI and related drought severity: an analysis using long-term data series. Water Resource Management, v. 30, p.5737-5757, 2016. https://doi.org/10.1007/s11269-016-1388-5.

PEREIRA, A. R.; VILLA NOVA, N. A.; SEDIYAMA, G. C. Evapotranspiração. Piracicaba: FEALQ, 1997.

PRUITT, W. O.; OETTINGEN, S. V.; MORGAN, D. L. Central California evapotranspiration frequencies. Journal of the Irrigation and Drainage Division, v. 98, p. 177-184, 1972.

RIBEIRO, A. M. A.; LUNARDI, D. M. C. A precipitação quinzenal provável para Londrina-PR, através da função gama. In: CONGRESSO BRASILEIRO DE AGROMETEOROLOGIA, 10., 1997, Piracicaba. Anais... Piracicaba: ESALQ, 1997. p. 95-97.

SAAD, J. C. C. Estudo das distribuições de frequência da evapotranspiração de referência e da precipitação pluvial para fins de dimensionamento de sistemas de irrigação. 1990. 124 f. Dissertação (Mestrado em Irrigação e Drenagem) - Escola Superior de Agricultura Luiz de Queiroz, Universidade de São Paulo, Piracicaba.

SAMPAIO, S. C.; QUEIROZ, M. M. F.; FRIGO, E. P.; LONGO, A. J.; SUSZEK, M. Estimativa e distribuição de precipitações decendiais para o Estado do Paraná. Irriga, v.12, p.38-53, 2007. https://doi.org/10.15809/irriga.2007v12n1p3853.

SEDIYAMA, G.; MELO, J. S. P.; ALVES, A. R.; COELHO, D.T. Determinação dos parâmetros da distribuição gama, em função das alturas médias mensais de precipitação dos dias chuvosos. Revista Ceres, v. 43, n. 247, p. 254-266, 1996.

SIMEPAR - SISTEMA METEOROLÓGICO DO PARANÁ. Solicitação de dados meteorológicos da região de Pinhais. SINEOAR, 2019. Disponível em < http://www.simepar.br/site_pw/faleconosco/da dosacademicos-create > Acesso em: 07 jul. 2019.

SOUZA, J. L. M. Fundamentos de matemática e estatística para formulação de modelos e análise de dados: aplicados às ciências agrárias. Curitiba, UFPR/SCA/DSEA/ Plataforma Moretti, 2018.

SOUZA, J. L. M. Ciclo da água na agricultura: fundamentos para o estudo do sistema solo-planta-atmosfera. Curitiba: UFPR/SCA/DSEA/Plataforma Moretti, 2017.

SOUZA, J. L. M.; JERSZURKI, D.; DAMAZIO, E. C. Relações funcionais entre precipitação provável e média em regiões e climas brasileiros. Pesquisa Agropecuária 


\section{Souza et al.}

Brasileira, v.48, p. 693-702, 2013.

THORNTHWAITE, C. W. An approach toward a rational classification of climate.

Geographical Review, New York, v. 38, n. 1, p. 55-94, 1948. 